Pro Windows PowerShell

Hristo Deshev 


\section{Pro Windows PowerShell}

\section{Copyright $\odot 2008$ by Hristo Deshev}

All rights reserved. No part of this work may be reproduced or transmitted in any form or by any means, electronic or mechanical, including photocopying, recording, or by any information storage or retrieval system, without the prior written permission of the copyright owner and the publisher.

ISBN-13 (pbk): 978-1-59059-940-2

ISBN-10 (pbk): 1-59059-940-3

ISBN-13 (electronic): 978-1-4302-0546-3

ISBN-10 (electronic): 1-4302-0546-6

Printed and bound in the United States of America 987654321

Trademarked names may appear in this book. Rather than use a trademark symbol with every occurrence of a trademarked name, we use the names only in an editorial fashion and to the benefit of the trademark owner, with no intention of infringement of the trademark.

Lead Editor: Tony Campbell

Technical Reviewer: Jon Rolfe

Editorial Board: Clay Andres, Steve Anglin, Ewan Buckingham, Tony Campbell, Gary Cornell, Jonathan Gennick, Kevin Goff, Matthew Moodie, Joseph Ottinger, Jeffrey Pepper, Frank Pohlmann, Ben Renow-Clarke, Dominic Shakeshaft, Matt Wade, Tom Welsh

Project Manager: Sofia Marchant

Copy Editor: Heather Lang

Associate Production Director: Kari Brooks-Copony

Production Editor: Laura Cheu

Compositor: Susan Glinert Stevens

Proofreader: Linda Seifert

Indexer: Julie Grady

Cover Designer: Kurt Krames

Manufacturing Director: Tom Debolski

Distributed to the book trade worldwide by Springer-Verlag New York, Inc., 233 Spring Street, 6th Floor, New York, NY 10013. Phone 1-800-SPRINGER, fax 201-348-4505, e-mail orders-ny@springer-sbm.com, or visit http://www. springeronline.com.

For information on translations, please contact Apress directly at 2855 Telegraph Avenue, Suite 600, Berkeley, CA 94705. Phone 510-549-5930, fax 510-549-5939, e-mail info@apress.com, or visit http://www. apress.com.

Apress and friends of ED books may be purchased in bulk for academic, corporate, or promotional use. eBook versions and licenses are also available for most titles. For more information, reference our Special Bulk Sales-eBook Licensing web page at http://www. apress.com/info/bulksales.

The information in this book is distributed on an "as is" basis, without warranty. Although every precaution has been taken in the preparation of this work, neither the author(s) nor Apress shall have any liability to any person or entity with respect to any loss or damage caused or alleged to be caused directly or indirectly by the information contained in this work.

The source code for this book is available to readers at http://www. apress.com. You will need to answer questions pertaining to this book in order to successfully download the code. 
To my wife, Yana, for all her love and support 


\section{Contents at a Glance}

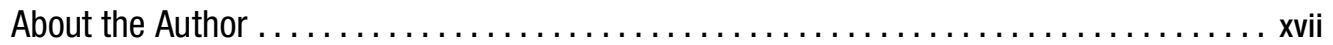

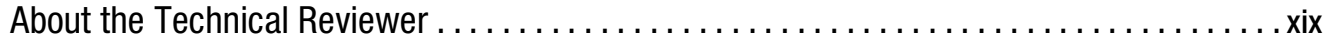

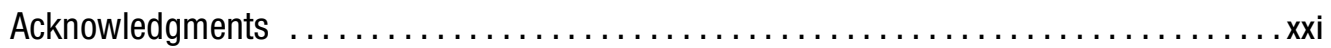

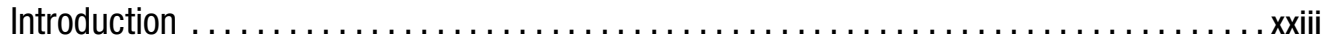

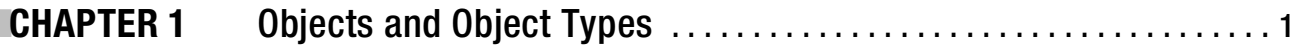

CHAPTER 2 Controlling Execution Flow $\ldots \ldots \ldots \ldots \ldots \ldots \ldots \ldots \ldots \ldots \ldots$

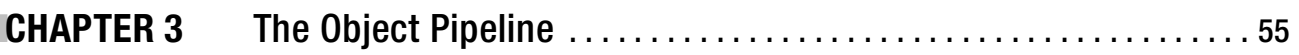

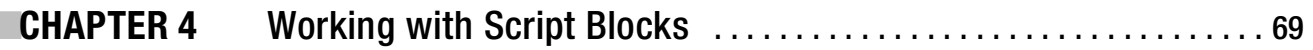

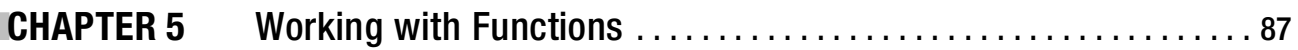

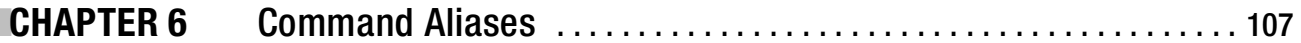

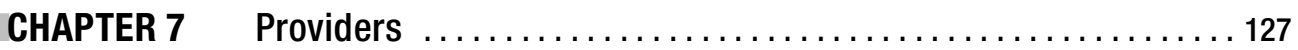

CHAPTER 8 Script Files . . . . . . . . . . . . . . . . . . . . . . . . 139

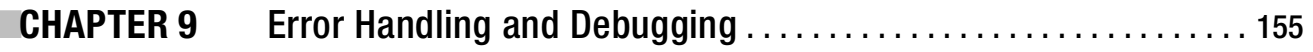

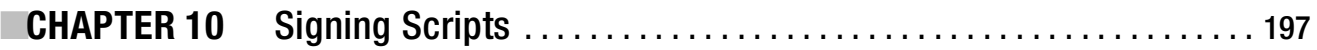

CHAPTER 11 The Shell Environment and Its Configuration $\ldots \ldots \ldots \ldots \ldots 219$

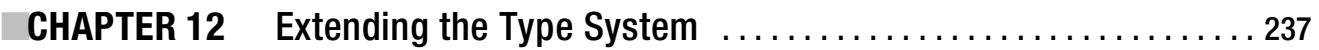

CHAPTER 13 Getting Help ...................................... 253

CHAPTER 14 Taming Processes and Services ..................... 275

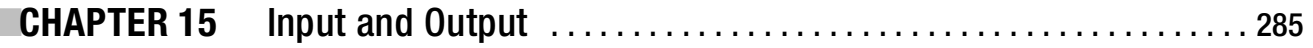

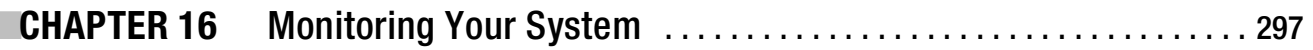

CHAPTER 17 PowerShell and the World Wide Web ................... 315

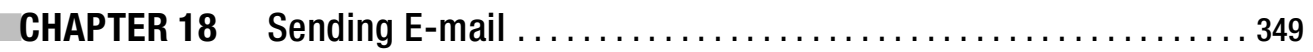

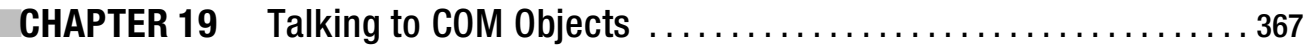

CHAPTER 20 Managing Windows with WMI $\ldots \ldots \ldots \ldots \ldots \ldots \ldots \ldots \ldots \ldots \ldots$

CHAPTER 21 PowerShell Community Extensions $\ldots \ldots \ldots \ldots \ldots \ldots \ldots \ldots . \ldots 4$ 
CHAPTER 22 PSEventing: .NET Events in PowerShell ................ 449

CHAPTER 23 Enhancing Tab Completion with PowerTab ................ 461

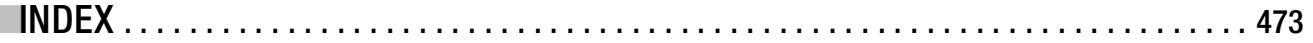




\section{Contents}

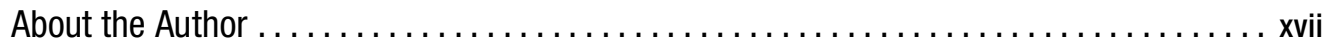

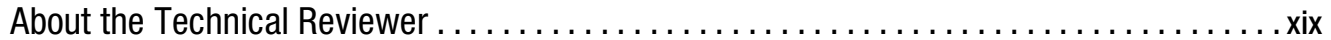

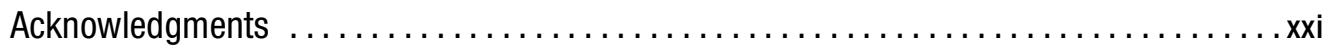

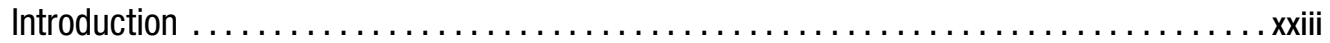

CHAPTER 1 Objects and Object Types $\ldots \ldots \ldots \ldots \ldots \ldots \ldots \ldots \ldots \ldots \ldots \ldots \ldots$

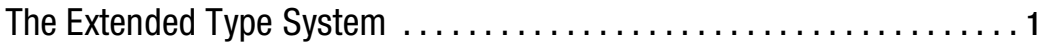

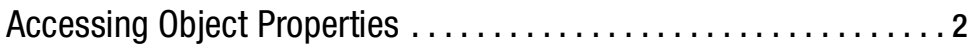

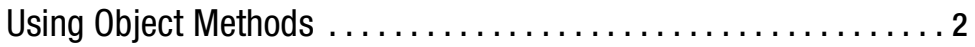

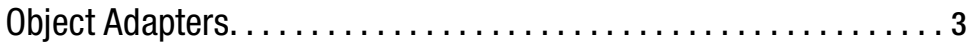

Type Extensions . ............................. 5

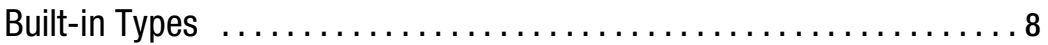

Strings and String 0 perations $\ldots \ldots \ldots \ldots \ldots \ldots \ldots \ldots \ldots, \ldots$

Numeric Types .................................... 14

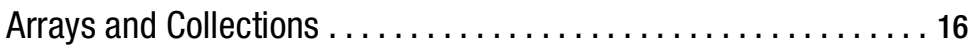

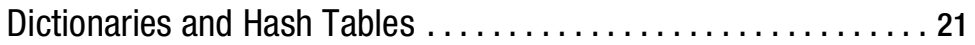

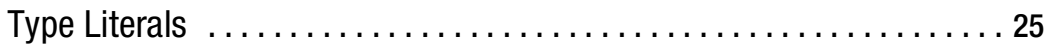

Type Conversion ............................. 26

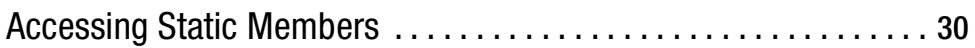

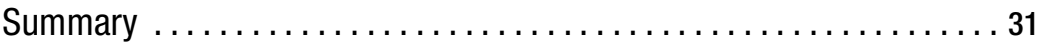

CHAPTER 2 Controlling Execution Flow $\ldots \ldots \ldots \ldots \ldots \ldots \ldots \ldots \ldots$

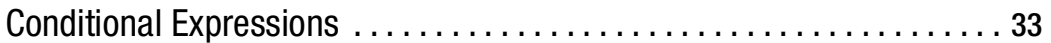

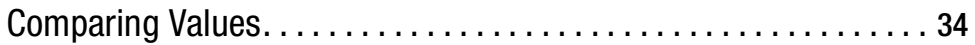

Implicit Type Conversions ......................... 36

Logic and Bitwise Operations ...................... 37

Boolean Conversions ............................ 41

The Power of String Conversions $\ldots \ldots \ldots \ldots \ldots \ldots \ldots \ldots \ldots 2$

Collections and Conditional Expressions ................ 43 


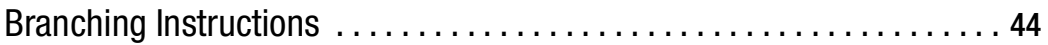

Simple Branching: if/else......................... 44

Multiple Branches and the switch Statement ............. 45

Loops: Repeatedly Executing Blocks of Code $\ldots \ldots \ldots \ldots \ldots \ldots \ldots 48$

The Simplest Loop: while $\ldots \ldots \ldots \ldots \ldots \ldots \ldots \ldots \ldots \ldots$

Loops and Counters: for Loop....................... 50

Executing Actions on All Objects in a Collection: foreach Loop. . . . 50

Controlling Loop Execution: break and continue Statements ..... 51

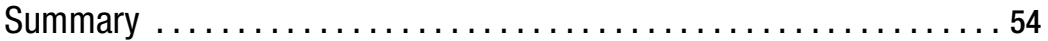

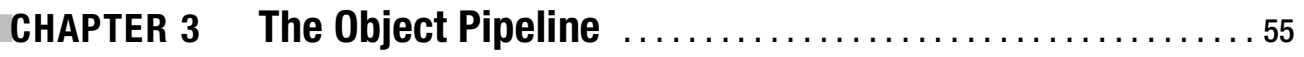

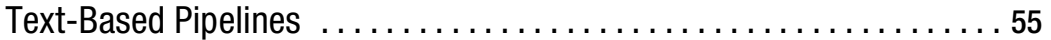

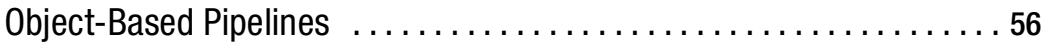

Processing Collections Using ForEach-Object $\ldots \ldots \ldots \ldots \ldots \ldots \ldots 59$

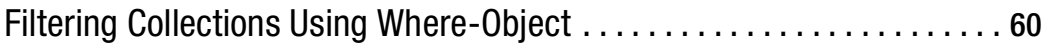

Adding or Deleting Properties Using Select-Object . . . . . . . . . . . 60

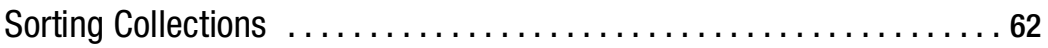

Pipeline Tees ..................................... 63

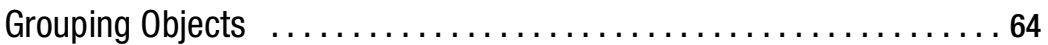

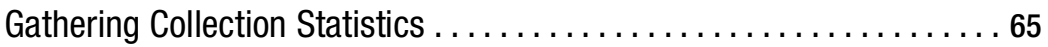

Detecting Changes and Differences Among Objects $\ldots \ldots \ldots \ldots \ldots 65$

The Object Pipeline and Functional Programming $\ldots \ldots \ldots \ldots \ldots \ldots 6$

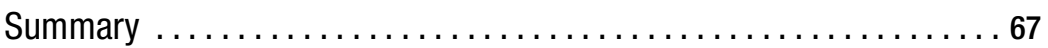

CHAPTER 4 Working with Script Blocks $\ldots \ldots \ldots \ldots \ldots \ldots \ldots \ldots \ldots$

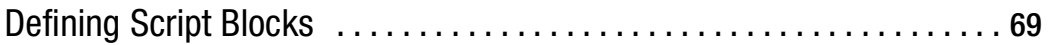

Passing Parameters and Returning Values $\ldots \ldots \ldots \ldots \ldots \ldots \ldots \ldots \ldots$

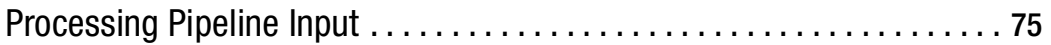

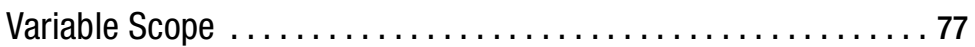

Invoking Strings as Expressions $\ldots \ldots \ldots \ldots \ldots \ldots \ldots \ldots \ldots \ldots$

Script Blocks as Delegates $\ldots \ldots \ldots \ldots \ldots \ldots \ldots \ldots \ldots \ldots \ldots . \ldots \ldots$

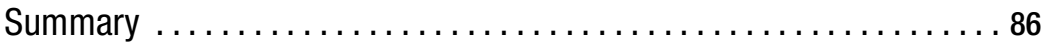

CHAPTER 5 Working with Functions $\ldots \ldots \ldots \ldots \ldots \ldots \ldots \ldots \ldots \ldots \ldots$

Defining Functions $\ldots \ldots \ldots \ldots \ldots \ldots \ldots \ldots \ldots \ldots \ldots \ldots$

Function Internals ........................... 88

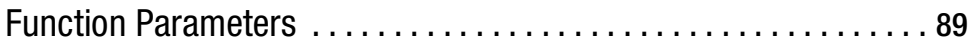

Passing Parameters by Reference $\ldots \ldots \ldots \ldots \ldots \ldots \ldots \ldots .91$ 
Returning Values............................ 93

Scoping Rules ............................... 94

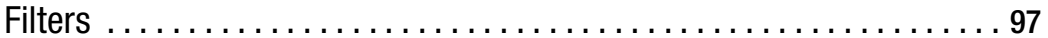

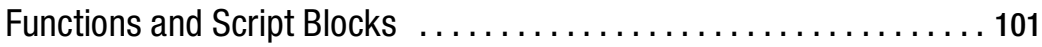

Implementing New Control Structures ................. 101

Script Blocks as Strategies ......................... 104

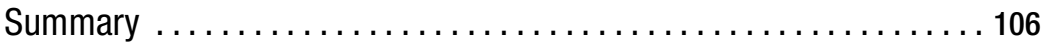

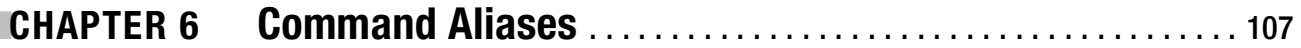

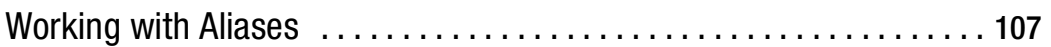

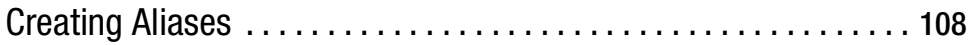

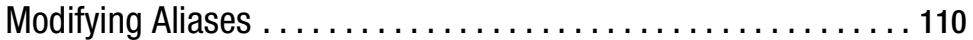

Exporting and Importing Aliases . . . . . . . . . . . . . . . . . 111

Aliasing Tips, Techniques, and Pitfalls .................... 115

Name Clashes.................................. 115

Complex Aliases . . . . . . . . . . . . . . . . . . . . . . 117

Removing Broken Aliases $\ldots \ldots \ldots \ldots \ldots \ldots \ldots \ldots \ldots \ldots \ldots 118$

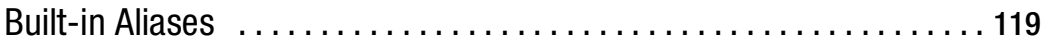

cmd.exe Look-alikes ........................... 120

UNIX Look-alikes............................... 121

Convenience Aliases. .......................... 123

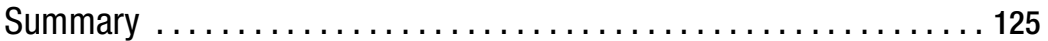

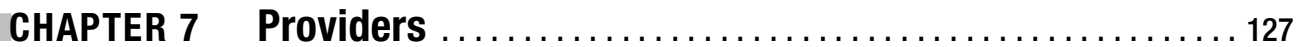

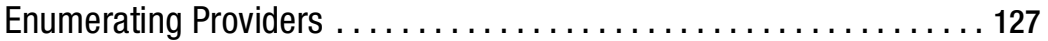

Drives .......................................... 129

Drive Scope ................................ 131

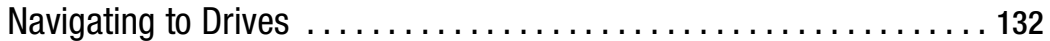

Provider Capabilities ............................... 134

Basic Provider Capabilities ......................... 134

Drive Providers . ............................... 134

Item Providers ................................... 134

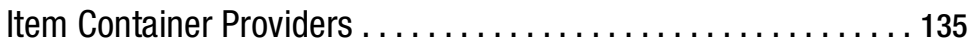

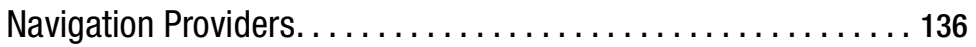

Item Content Providers.......................... 136

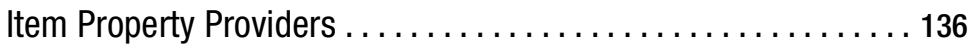

Dynamic Item Property Providers $\ldots \ldots \ldots \ldots \ldots \ldots \ldots \ldots \ldots \ldots \ldots$

Item Security Descriptor Providers . . . . . . . . . . . . . . . 137

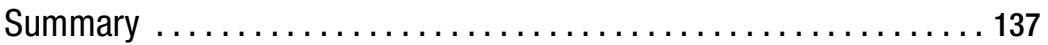




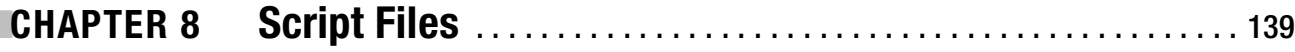

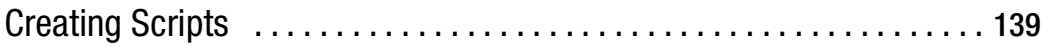

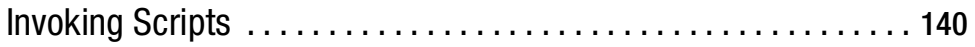

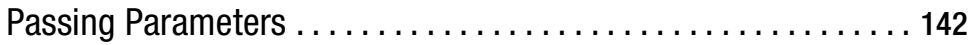

Returning Values............................. 146

Executing PowerShell Scripts from 0ther Environments .......... 148

Developing and Maintaining Script Libraries ................. 149

Dot-sourcing as a Means to Include Script Libraries ......... 150

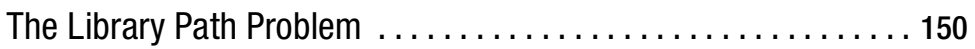

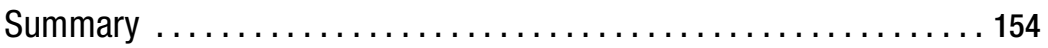

CHAPTER 9 Error Handling and Debugging $\ldots \ldots \ldots \ldots \ldots \ldots \ldots \ldots$

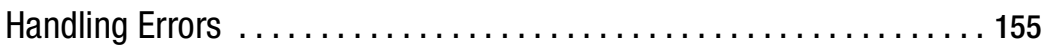

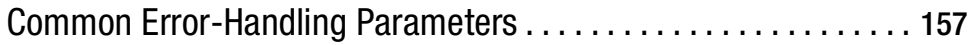

Trapping Errors . ............................. 160

Capturing Nonterminating Errors $\ldots \ldots \ldots \ldots \ldots \ldots \ldots \ldots \ldots$

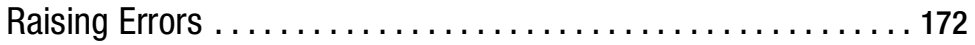

Debugging Your Code $\ldots \ldots \ldots \ldots \ldots \ldots \ldots \ldots \ldots \ldots \ldots \ldots \ldots \ldots \ldots \ldots$

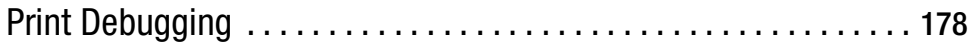

Stepping Through Scripts and Breaking Execution. ........ 188

Tracing Script Execution Details . . . . . . . . . . . . . . . 190

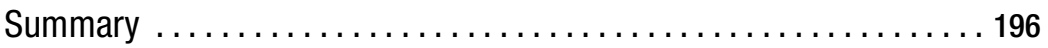

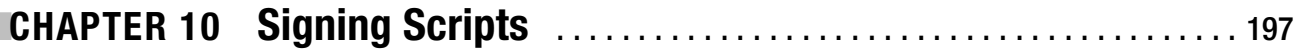

How Code Signing Works ......................... 197

Guaranteeing Script Integrity. $\ldots \ldots \ldots \ldots \ldots \ldots \ldots \ldots \ldots \ldots$

Certifying the Origin of a Script. . . . . . . . . . . . . . . . 198

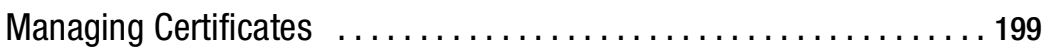

Creating a Self-Signed Certificate ...................... 202

Creating a Certification Authority Certificate ............. 202

Issuing a Code-Signing Certificate $\ldots \ldots \ldots \ldots \ldots \ldots \ldots \ldots 206$

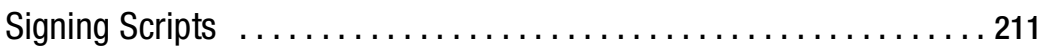

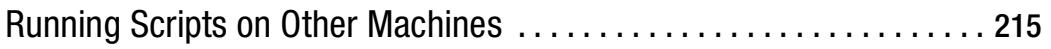

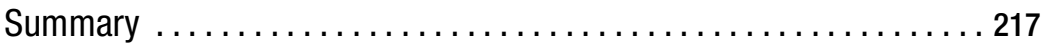


Shell Hosts ................................... 219

User Profile Scripts . ............................. 223

Settings for All Users and All Shells . ................. 223

Settings for All Users Using a Specific Shell . . . . . . . . . . . . 224

Settings for a Specific User and All Shells ................ 224

Settings for a Specific User and a Specific Shell ............ 224

Working with Saved Console Settings $\ldots \ldots \ldots \ldots \ldots \ldots \ldots \ldots \ldots . \ldots \ldots$

Changing the Prompt Settings . . . . . . . . . . . . . . . . . . 228

Tab Expansion: How Command Completion Works $\ldots \ldots \ldots \ldots \ldots 231$

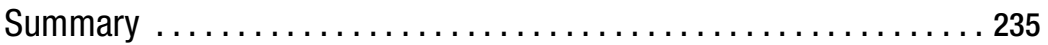

CHAPTER 12 Extending the Type System ................... 237

Modifying Objects and Types $\ldots \ldots \ldots \ldots \ldots \ldots \ldots \ldots \ldots \ldots \ldots \ldots \ldots \ldots \ldots$

Adding Members to Single 0bjects.................. 238

Adding Members to All Instances of a Class .............. 244

Extending Object Formatting .......................... 247

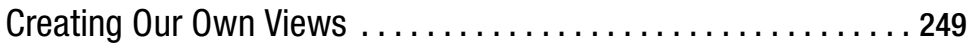

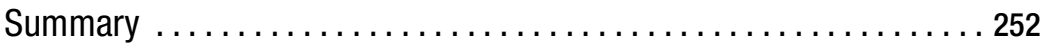

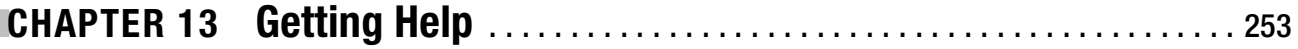

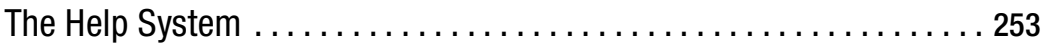

The Basics.................................. 254

Parameter Details............................. 256

Advanced Techniques .......................... 258

Getting Command Information $\ldots \ldots \ldots \ldots \ldots \ldots \ldots \ldots \ldots \ldots \ldots \ldots$

Getting Information About Objects $\ldots \ldots \ldots \ldots \ldots \ldots \ldots \ldots \ldots \ldots \ldots$

Using the Internet to Get Help $\ldots \ldots \ldots \ldots \ldots \ldots \ldots \ldots \ldots \ldots . \ldots \ldots$

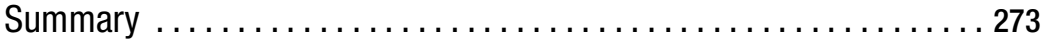

CHAPTER 14 Taming Processes and Services $\ldots \ldots \ldots \ldots \ldots \ldots \ldots \ldots$

Working with Processes ........................... 275

Listing Processes: Getting the Big Picture $\ldots \ldots \ldots \ldots \ldots \ldots 276$

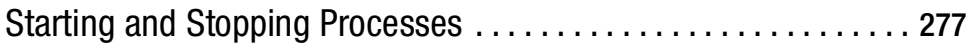

Processes and Their Windows .................... 278

Process Modules: On What Libraries Does This

Baby Depend? ........................... 279 
Getting Information About a Program's Publisher......... 280

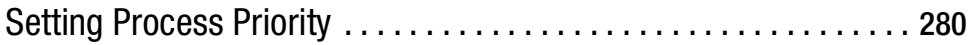

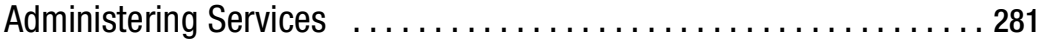

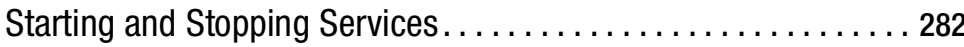

Configuring Services $\ldots \ldots \ldots \ldots \ldots \ldots \ldots \ldots \ldots \ldots \ldots \ldots \ldots \ldots$

Analyzing Service Dependencies .................... 283

Summary ...................................... 284

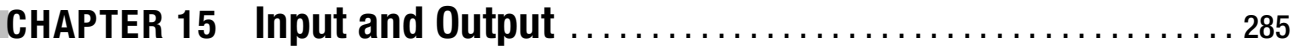

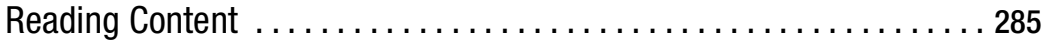

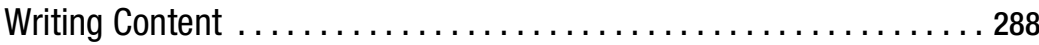

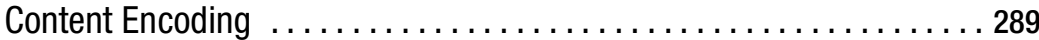

Getting Byte Content. ......................... 290

Different Unicode Encodings . . . . . . . . . . . . . . . . . . 291

Extracting Data from Text ........................... 294

Finding Matches for a Regular Expression. .............. 294

Finding String 0ccurrences Inside Files............... 295

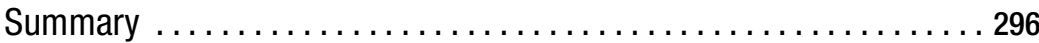

CHAPTER 16 Monitoring Your System .......................... 297

Working with the Windows Event $\log \ldots \ldots \ldots \ldots \ldots \ldots \ldots \ldots . \ldots \ldots 7$

Reading from the System Event Logs $\ldots \ldots \ldots \ldots \ldots \ldots \ldots . \ldots 298$

Writing to the Event Log......................... 302

Performance Counters ............................... 306

Consuming Counter Data.......................... 307

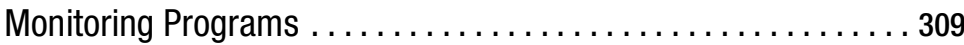

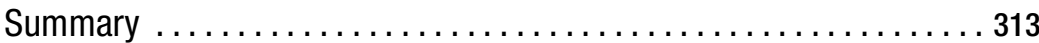

CHAPTER 17 PowerShell and the World Wide Web $\ldots \ldots \ldots \ldots \ldots \ldots$

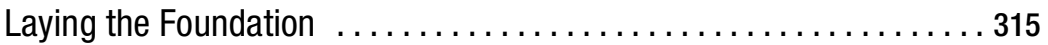

Fetching Files from the Web ....................... 316

Setting Connection Options and Debugging

Connection Problems .......................... 318

Testing and Validating Web Sites ....................... 328

Test-Url: Verifying a Page's Existence ................... 328

Checking If a Page Contains Broken Links .............. 330 


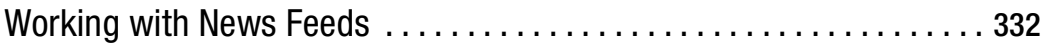

Getting Feeds ................................. 333

Calling Web Services ................................ 338

Calling Web Services Using HTTP GET Requests . . . . . . . . 338

Calling Web Services Using HTTP POST Requests . . . . . . . . . 340

Calling Web Services Using the SOAP Protocol. . . . . . . . . . . 342

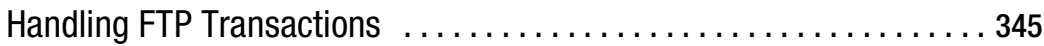

Downloading Files from an FTP Server ................ 345

Uploading Files to an FTP Server . . . . . . . . . . . . . . . . 346

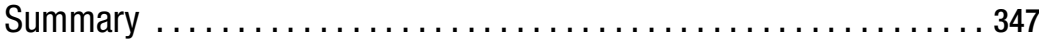

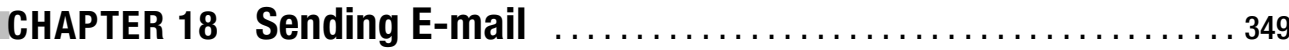

Using System.Net.Mail . .......................... 349

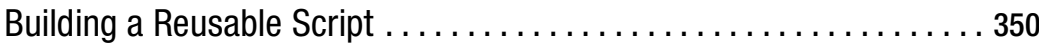

Configuring Recipients ................................ 352

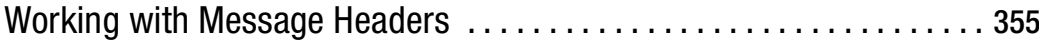

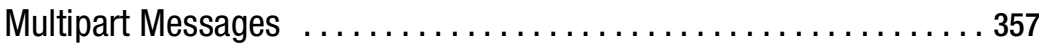

Message Views ................................... 357

Attaching Files to Messages . . . . . . . . . . . . . . . . . 359

Embedding Media in Messages . ..................... 361

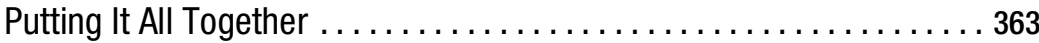

Summary .......................................... 365

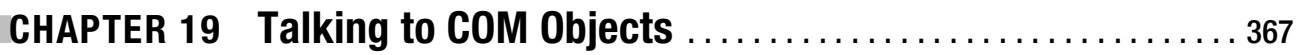

How COM Works $\ldots \ldots \ldots \ldots \ldots \ldots \ldots \ldots \ldots \ldots \ldots, \ldots, \ldots, \ldots, \ldots, \ldots$

How PowerShell Supports COM $\ldots \ldots \ldots \ldots \ldots \ldots \ldots \ldots \ldots \ldots . \ldots \ldots$

Scripting Programs Through Their COM Interfaces . . . . . . . . . . 369

Automating Microsoft Word . . . . . . . . . . . . . . . . . . . . 369

Scripting Microsoft Excel $\ldots \ldots \ldots \ldots \ldots \ldots \ldots \ldots \ldots \ldots \ldots \ldots$

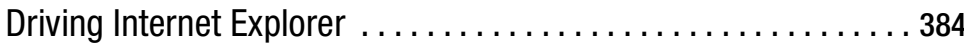

Windows Script Host Code Interoperability . . . . . . . . . . . . . 389

Evaluating Code ............................... 390

Exposing Objects from MSScriptControl $\ldots \ldots \ldots \ldots \ldots \ldots \ldots . \ldots . \ldots 2$

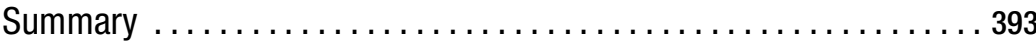




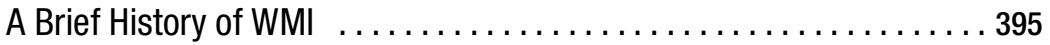

WBEM and WMI Components $\ldots \ldots \ldots \ldots \ldots \ldots \ldots \ldots \ldots \ldots \ldots \ldots \ldots$

The Common Information Model. ................... 396

Network Transport ............................. 397

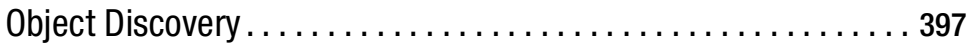

PowerShell's WMI Support $\ldots \ldots \ldots \ldots \ldots \ldots \ldots \ldots \ldots \ldots \ldots \ldots . \ldots \ldots$

Get-WmiObject: The WMI Query Tool. . . . . . . . . . . . . . 399

Language Support for WMI 0bjects $\ldots \ldots \ldots \ldots \ldots \ldots \ldots \ldots \ldots \ldots$. 401

Exploring CIM Classes and 0bjects $\ldots \ldots \ldots \ldots \ldots \ldots \ldots \ldots . \ldots 404$

Getting a List of Classes and Namespaces . . . . . . . . . . . . 404

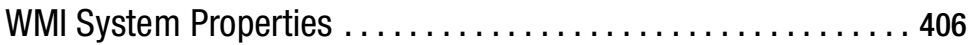

Querying Hardware Devices $\ldots \ldots \ldots \ldots \ldots \ldots \ldots \ldots \ldots \ldots \ldots \ldots$

Getting Information About Software $\ldots \ldots \ldots \ldots \ldots \ldots \ldots \ldots \ldots, 408$

Operating System Updates . . . . . . . . . . . . . . . . . . 408

Installed Programs ............................ 409

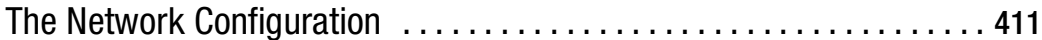

Generating WMI Code ............................... 411

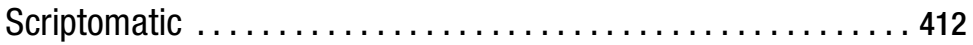

Summary $\ldots \ldots \ldots \ldots \ldots \ldots \ldots \ldots \ldots \ldots \ldots \ldots \ldots \ldots \ldots \ldots, 414$

CHAPTER 21 PowerShell Community Extensions $\ldots \ldots \ldots \ldots \ldots \ldots 417$

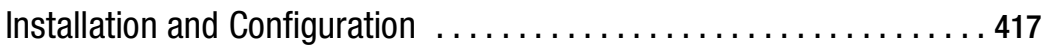

Tab Expansion................................. 418

Editing Configuration Files...................... 418

Getting Help............................... 419

File System Utilities $\ldots \ldots \ldots \ldots \ldots \ldots \ldots \ldots \ldots \ldots \ldots \ldots \ldots, 421$

Navigation Helpers $\ldots \ldots \ldots \ldots \ldots \ldots \ldots \ldots \ldots \ldots \ldots \ldots \ldots, \ldots 21$

NTFS Helpers. . . . . . . . . . . . . . . . . . . . . . 422

Compressing and Archiving Files ................... 424

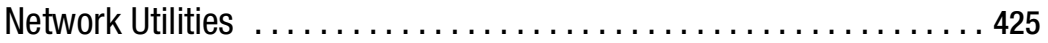

Executing Processes and Commands $\ldots \ldots \ldots \ldots \ldots \ldots \ldots \ldots 427$

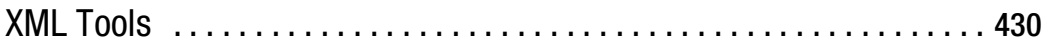

Working with Image Files .......................... 435

Clipboard Helpers $\ldots \ldots \ldots \ldots \ldots \ldots \ldots \ldots \ldots \ldots \ldots \ldots \ldots \ldots \ldots \ldots \ldots \ldots$ 


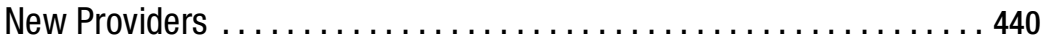

Reading from the .NET Global Assembly Cache ........... 440

Exploiting the Internet Explorer Feed Store . . .............. 441

Manipulating Windows Active Directory $\ldots \ldots \ldots \ldots \ldots \ldots \ldots 443$

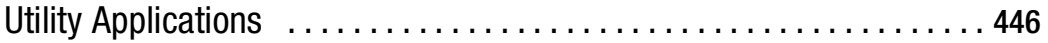

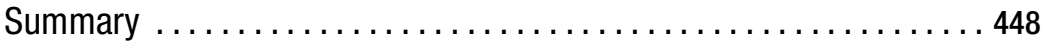

CHAPTER 22 PSEventing: .NET Events in PowerShell $\ldots \ldots \ldots \ldots \ldots 449$

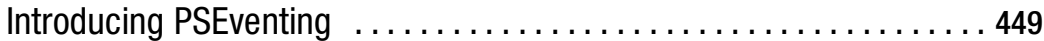

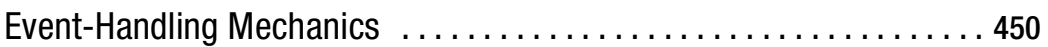

FileSystemWatcher: A Real-World Example $\ldots \ldots \ldots \ldots \ldots \ldots \ldots 451$

Monitoring Entries Written to the System Event Logs $\ldots \ldots \ldots \ldots . .452$

Handling WMI Events ............................. 454

Detecting If Our Script Has Been Terminated by the User $\ldots \ldots \ldots 456$

Using Script Blocks As Event Handlers $\ldots \ldots \ldots \ldots \ldots \ldots \ldots \ldots 457$

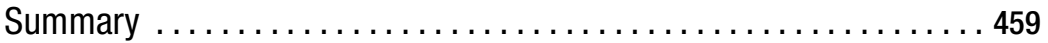

CHAPTER 23 Enhancing Tab Completion with PowerTab .......... 461

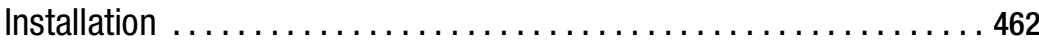

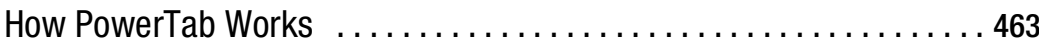

Data Grid Pop-up Windows ....................... 466

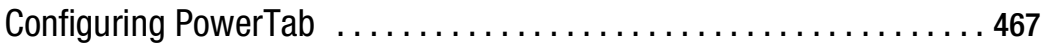

The IntelliSense Completion Handler . . . . . . . . . . . . . 468

The Tab Expansion Database . ..................... 470

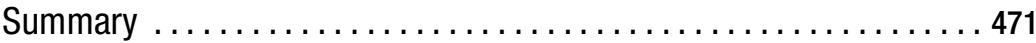

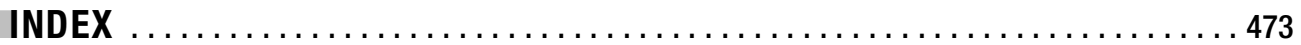




\section{About the Author}

HRISTO DESHEV has been a software developer, a team leader, and a product manager for Telerik, the leading vendor of user interface components (http://www.telerik.com). For the past several years, his focus has been on creating rich and, at the same time, robust and reliable web and desktop components running on the Microsoft .NET platform. A major goal for him and his team is to predictably deliver working software-applying agile software engineering practices to create rock-solid solutions targeting all modern web browsers and supporting multiple Visual Studio .NET versions.

Hristo is a reformed UNIX fanatic who now runs Windows and tries to apply his scripting skills and experiences to the Microsoft platform. He is also a tool freak: anything that can help automate a task - be it a scriptable utility or a full-blown scripting language-is a welcome addition to his toolbox. PowerShell has been a passion of his since its early unofficial releases due to its ease of use and almost incredible ability to automate all Windows-related tasks without needing other tools.

You can reach Hristo through his blog at http://weblogs.asp. net/hristodeshev/. 


\section{About the Technical Reviewer}

JON ROLFE has been an IT professional for more than 12 years and is currently a senior solutions architect for one of the world's largest IT services companies. He specializes in designing secure Microsoft-based enterprise architectures for clients in the United Kingdom's public sector and large blue-chip companies. During the course of his career, he has gained extensive experience at all stages of the IT life cycle, including enterprise architecture design, software development, rapid system deployment, and large enterprise systems support. Although he specializes in Microsoft technologies, Jon also has an interest in other technologies including Linux and wrote the book Using Ubuntu Linux (Bernard Babani, 2007).

When not working, Jon has an active interest in digital photography, follows motor sports, and enjoys mountain biking and skiing. He can be contacted through his web site at http://JonRolfe.com. 


\section{Acknowledgments}

still find it hard to believe that I wrote an entire book! It has been an enjoyable project that was also quite formidable and downright hard. I would never have done it all on my own, without the huge amounts of help I got from many people.

First, I would like to thank the entire Apress team that guided me through the process: Tony Campbell for providing important input and valuable feedback on the content, Sofia Marchant for being so flexible with my sometimes late chapters, Heather Lang for her extraordinary ability to turn my often unreadable writings into well-thought-out text. Jon Rolfe deserves a special mention here; he has been an awesome technical reviewer. He not only spotted a host of problems with the sample scripts but was keen to provide solutions and improvement ideas; thus his efforts became one of the most important factors in getting this book done.

I would also like to thank the Microsoft PowerShell team for creating such a wonderful product. Being a long-time UNIX user, I have been disappointed by the primitive Windows command line shell. With PowerShell, this is no longer the case.

The PowerShell community deserves special recognition too. The wealth of information and thoughtful discussions on blogs and online forums has been an incredible inspiration when writing this book.

Last, but not least, I am personally thankful to the people behind the open source tools covered in the book: Keith Hill and the numerous contributors to the PowerShell Community Extensions project; Marc van Orsouw, the creator of the PowerTab tab completion extension; and Oisin Grehan, the man behind the PowerShell Eventing Library and many other PowerShellrelated open source projects. You guys rock! 


\section{Introduction}

remember the first time I got to play with Windows PowerShell—I had some spare time on my hands, and after reading a blog post, I quickly set off to download the unofficial pre-release bits that were available at the time. I originally had very low expectations about the tool—all I wanted was a shell that is up to par with its UNIX cousins. Boy, was I wrong! The moment I learned about object pipelines, I knew this tool was going to change the way I think about automating tasks on Windows. Fast forward a couple of months, and here I am, so much in love with PowerShell that I even wrote a whopping 400-page book about it.

\section{Why Another Book on Windows PowerShell?}

As much as I like PowerShell, its plain-console looks make it very easy for people to dismiss it without really seeing what lies beneath the surface. I feel it is still largely undiscovered-I know many professionals who have yet to try it out. When administrators see PowerShell for the first time, many simply shrug, assuming it's just another version of the primitive command prompt we inherited from DOS.

The most successful way for me to recruit a new person into the ranks of happy PowerShell users is by example. When presented with an automation problem, my first suggestion is, "Let's try to do it in PowerShell." A couple of lines later, the usual reaction is, "Wow, can it really do that?" A short and sweet command can win many hearts for PowerShell. Hopefully, the examples in this book will win your heart too-it is my goal to share with you the joy of working with a modern automation environment that will change your professional life forever.

\section{What's in This Book?}

When working on the book, I separated it into sections, and this distinction may be helpful to you too.

The first section includes Chapters 1 through 14. It introduces the shell's object-oriented features and its means for providing basic abstraction like script blocks, functions, scripts, aliases, and providers. It is best to read those chapters in sequence to get a solid understanding of the advanced techniques that allow you to work with the shell's type system, get to know its security infrastructure, learn about the documentation facilities, and start using important debugging techniques.

The second section spans Chapters 15 to 21. The text uses advanced scripting techniques to work with .NET, COM, and WMI objects. This section's goal is twofold. On one hand, you'll learn how to work with external code and employ those techniques to automate almost any task on a Windows system. On the other hand, you are solving real-world problems that are very likely to land on your plate some time soon. The chapters describe how to start and stop processes 
and services, work with text-based I/O, use performance counters to monitor your system, download and upload files to the web, send e-mail, automate programs via their COM interfaces, and get information and manage operating system objects through WMI. The chapters can be read in random order, and you can easily use them for reference.

The last section of the book is about tools and add-ons that you can use to extend the shell and become even more productive. Many programs can get much easier to use after extending them with a tool that provides an important missing feature. The book shows how you can use freely available open source tools to boost your productivity. An important part of my secret in winning over new people for PowerShell lies in the fact that I always do my demonstrations with PowerShell Community Extensions and PowerTab installed. Those two tools are described in Chapters 21 and 23 respectively.

\section{Do I Need Prior Programming Experience?}

The book is written by a programmer-does that mean you need to be a programmer to read it? Not at all! I have done my best to make the text easy to digest for just about anyone with a little prior scripting experience. The PowerShell scripting language bears a lot of similarities to other scripting languages, and users will be able to draw parallels between it and VBScript, JScript, batch scripts, UNIX shell scripts, and maybe even Perl or Python. People new to scripting should be able to just use any of the working code samples as they are, without needing to learn the details or any of the inner workings of the code. 